\title{
i-Open Sets in Bitopological Spaces
}

Amir A. Mohammed

amirabdulillah64@gmail.com
Sabeeh W. Askandar

sabeehqaqus@yahoo.com

Department of Mathematics

College of Education

University of Mosul, Mosul, Iraq

\section{Received on: $27 / 06 / 2013$}

\section{ABSTRACT}

Accepted on: 11/11/2013

In this paper, we defined $\mathrm{i}$-open sets and $\mathrm{i}$-star generalized $\mathrm{w}$-closed sets in bitopological spaces $\left(X, \tau_{1}, \tau_{2}\right)$ by using the definition of i-open sets in topological space $(X, \tau)$ (see[6]). We present some fundamental properties and relations between these classes of sets, further we give examples to explain these relations.

Keywords: i-open sets, bitopological spaces.

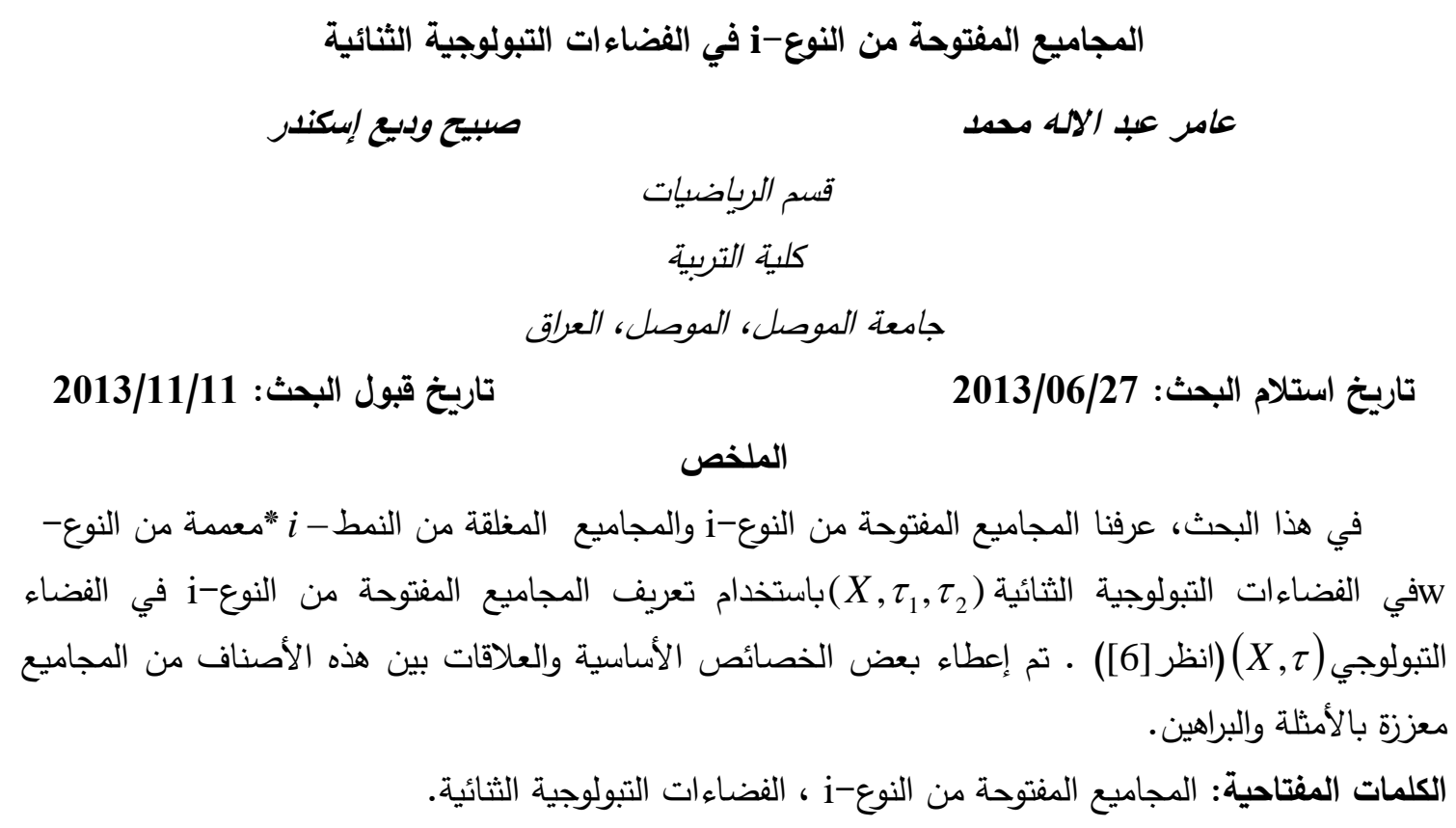

\section{Introduction}

Sheik and Sundaram in 2004 [9], introduced $\mathrm{g} *$-closed sets in bitopological spaces. Kannan and Chandrasekhara in 2006 [4], introduced regular star generalized closed sets in bitopological spaces. Mahdi in 2007 [5], introduced the concept of semi-open and semi-closed sets in bitopological spaces. Benchalli, Patil and Rayanagoudar in 2010 [2], introduced w-locally closed sets in bitopological spaces. Sheik and Maragathavalli in 2010 [8], introduced the concept of strongly $\alpha g *$ - closed sets in bitopological spaces. Nagaveni and Rajarubi in 2012 [7], introduced GRW-closed sets and GRW-continuity in bitopological spaces. Mohammed and Askandar In 2012 [6], introduced the concept of i-open sets as: A subset $A$ of a topological space $(X, \tau)$ is said to be i-open set[6] if there exists an open set $G \neq \phi, X$ such that $\mathrm{A} \subseteq C l(\mathrm{~A} \cap \mathrm{G})$. The complement of an i-open set is called $\mathrm{i}$-closed set, which could entire them together with many other concepts of generalized open sets. The aim of this paper is to introduce the concept of $i$-open sets in bitopological spaces $\left(X, \tau_{1}, \tau_{2}\right)$. This class of sets may be to enter together with other classes of sets in bitopological spaces which have been mentioned above for comparison and to find the similar properties and characterizations. Throughout this 
work, $\tau^{i}$ is a family of all $\mathrm{i}$-open sets[6] of $\mathrm{X}$ ). This work consists of two sections. In the first one, we define i-open sets in bitopological spaces and we give many related examples. In the second section, we define i-star generalized $w$-closed sets, i-star generalized $w$-open sets and study their basic properties in bitopological spaces. $\left(X, \tau_{1}, \tau_{2}\right)$ denote a bitopological space, where $\left(X, \tau_{1}\right)$ and $\left(X, \tau_{2}\right)$ are topological spaces. For any subset $A \subseteq X, \tau_{i}-\operatorname{Int}(A)$ and $\tau_{i}-C l(A)$ denote the interior and closure of a set $A$ with respect to the topology $\tau_{i}$. A point $x \in X$ is called a condensation point of $A$ [3] if for each $U \in \tau$ with $x \in U$, the set $U \cap A$ is uncountable. $A$ is called $w$-closed [3] if it contains all its condensation points. The complement of an $w$-closed set is called $w$-open. The $w$-closure [3] and $w$-int erior [3] of $A$ with respect to the topology $\tau_{i}$, that can be defined in a manner similar to $\tau_{i}-C l(A)$ and $\tau_{i}-\operatorname{int}(A), \quad$ respectively, will be denoted by $\tau_{i}-C l_{w}(A)$ and $\tau_{i}-\operatorname{int}_{w}(A)$, respectively. $A^{C}$ denotes the complement of $A$ in $X$.

\section{1. i-Open Sets in Bitopological Spaces.}

In this section, we define i-open sets in bitopological spaces by giving many related examples and we study the properties of these sets. Also we define many concepts of generalized open sets in bitopological spaces and we give many related examples.

Definition 1.1. Let $\left(X, \tau_{1}, \tau_{2}\right)$ be a bitopological space, a subset $\mathrm{A}$ of $\mathrm{X}$ is said to be $\left(\tau_{1} \tau_{2}-i\right.$-open set $)$ if there exists $\tau_{1}$-open set $U \neq \phi, X$ s.t. $A \subseteq \tau_{2}-C l(A \cap U)$. The complement of ( $\tau_{1} \tau_{2}-i$-open set $)$ is called $\left(\tau_{1} \tau_{2}-i\right.$-closed set $)$.

Definition 1.2. A bitopological space $\left(X, \tau_{1}, \tau_{2}\right)$ is said to be Bi-Topologically Extended for i-open sets (Bi.T.E.I.) if $\left(X, \tau_{1} \tau_{2}-i\right.$-open sets) is a topological space. On the other hand, if $\left(X, \tau_{1} \tau_{2}-i\right.$-open sets $)$ is not a topological space then, $\left(X, \tau_{1}, \tau_{2}\right)$ is called non-Bi-Topologically Extended for i-open sets(not Bi.T.E.I. ). Where, $\tau_{1} \tau_{2}-i$-open sets denote the family of all $\mathrm{i}$-open sets in the bitopological space $\left(X, \tau_{1}, \tau_{2}\right)$.

Example 1.3. Let $X=\{a, b, c\}, \tau_{1}=\{\phi,\{a\}, X\}, \tau_{2}=\{\phi,\{a\},\{a, b\}, X\}$.

$\tau_{1}$-open sets are $: \phi,\{a\}, X . \tau_{2}$-closed sets are $: \phi,\{b, c\},\{c\}, X$.

$\{a\} \subset\left(\tau_{2}-C l(\{a\} \cap\{a\})=X\right),\{a, b\} \subset\left(\tau_{2}-C l(\{a, b\} \cap\{a\})=X\right)$

$\{a, c\} \subset\left(\tau_{2}-C l(\{a, c\} \cap\{a\})=X\right)$.

Then, $\{a\},\{a, b\},\{a, c\}$ are $\tau_{1} \tau_{2}-i$-opensets.

But, $\{b\},\{c\},\{b, c\}$ are not $\tau_{1} \tau_{2}-i$-opensetsbecause there is no existence $\tau_{1}-$ open set $U$ s.t. $\{b\} \subset\left(\tau_{2}-C l(\{b\} \cap U)\right),\{c\} \subset\left(\tau_{2}-C l(\{c\} \cap U)\right)$ $\{b, c\} \subset\left(\tau_{2}-C l(\{b, c\} \cap U)\right)$ Therefore, $\tau_{1} \tau_{2}-i$-open sets $=\{\phi,\{a\},\{a, b\},\{a, c\}, X\}$. $\tau_{1} \tau_{2}-i$-closed sets $=\phi,\{b, c\},\{c\},\{b\}, X$ Where, $\left(X, \tau_{1} \tau_{2}-i\right.$-open sets $)$ is a topological space. Then, $\left(X, \tau_{1}, \tau_{2}\right)$ is a Bi.T.E.I. space.

Example 1.4. Let $X=\{a, b, c, d\}, \tau_{1}=\{\phi,\{a\},\{b, c, d\} X\}, \tau_{2}=\{\phi,\{a\},\{c\},\{a, c\}, X\}$. 
$\tau_{1}$-open sets are $: \phi,\{a\},\{b, c, d\}, X$.

$\tau_{2}$-closed sets are $: \phi,\{b, c, d\},\{a, b, d\},\{b, d\}, X$.

By the same way, in Example 1.3, we have:

$\tau_{1} \tau_{2}-i$-open sets $=\{\phi,\{a\},\{b\},\{c\},\{d\},\{b, c\},\{b, d\},\{c, d\}$,

$\{b, c, d\},\{a, b\},\{a, d\},\{a, b, d\}, X\}$

$\tau_{1} \tau_{2}-i-$ closed sets $=\{\phi,\{b, c, d\},\{a, c, d\},\{a, b, d\},\{a, c, d\},\{a, d\}$,

$\{a, c\},\{a, b\},\{a\},\{c, d\},\{b, c\},\{c\}, X\}$.

Where, $\left(X, \tau_{1} \tau_{2}-i\right.$-open sets $)$ is not a topological space. Then, $\left(X, \tau_{1}, \tau_{2}\right)$ is not Bi.T.E.I. space.

Definition 1.5. Let $\left(X, \tau^{i}\right)$ be a topological space and let $A$ be a subset of $X$. Recall that the intersection of all $\mathrm{i}$-closed sets containing $A$ is called i-closure of $A[6]$, denoted by $C l_{i}(A): C l_{i}(A)=\bigcap_{i \in \Lambda} F_{i} \cdot A \subseteq F_{i} \quad \forall i$ where, $F_{i}$ is i-closed set $\forall i$ in a topological space $(X$, $\left.\tau^{i}\right) . C l_{i}(A)$ is the smallest $\mathrm{i}$-closed set containing $A$.

Definition 1.6. Let $\left(X, \tau^{i}\right)$ be a topological space and let $A$ be a subset of X. Recall that the union of all i-open sets contained in $A$ is called i-Interior of $A[6]$, denoted by $\operatorname{Int}_{i}(A)$. $\operatorname{Int}_{i}(A)=\bigcup_{i \in \Lambda} I_{i} \quad I \subseteq A \quad \forall i$. Where, $I_{\mathrm{i}}$ is i-open set $\forall i$ in a topological space $\left(X, \tau^{i}\right)$. $\operatorname{Int}_{i}(A)$ is the largest $\mathrm{i}$-open set contained in $A$.

Theorem 1.7. Every $\tau_{1}$-open set is $\left(X, \tau_{1}, \tau_{2}\right)$ in $i$-openset

$\operatorname{Or}\left(\tau_{1} \subset\left(\tau_{1} \tau_{2}-i\right.\right.$-open sets $\left.)\right)$.

Proof Let $X$ be a finite non empty set. Let $\tau_{1}=\left\{\phi, A_{1}, A_{2}, \ldots \ldots A_{n}, X\right\}, \tau_{2}=\left\{\phi, B_{1}, B_{2}, \ldots \ldots ., B_{n}, X\right\}$.

Where, $A_{i} \subset X, B_{i} \subset X \forall i$.

$\tau_{1}$-open sets are : $\phi, A_{1}, A_{2}, \ldots \ldots . A_{n}, X$.

$\tau_{2}$-closed sets are $: \phi, X-B_{1}, X-B_{2}, \ldots \ldots . ., X-B_{n}, X$.

$\tau_{2}-C l\left(A_{i} \cap A_{i}\right)=\bigcap_{A_{i} \cap A_{i} \subset F}$, where $F$ is $\tau_{2}$-closed set.

At least, $\mathrm{X}$ is a $\tau_{2}-$ closed set contains $A_{i} \cap A_{i} \forall i$.

Hence, $\tau_{2}-C l\left(A_{i} \cap A_{i}\right)=\bigcap_{A_{i} \cap A_{i} \subset F}=X$.

Therefore, $A_{i} \subset\left(\tau_{2}-C l\left(A_{i} \cap A_{i}\right)=\bigcap_{A_{i} \cap A_{i} \subset F} F=X\right) \forall i$.

Then, $\left(\tau_{1} \subset\left(\tau_{1} \tau_{2}-i\right.\right.$-open sets $\left.)\right)$.

The converse of Theorem 1.7 is not true. Indeed, in Example $1.4\{b, c\}$ is $\tau_{1} \tau_{2}-i$-open set, but is not $\tau_{1}$-open set.

Definition 1.8. Let $(X, \tau)$ be a topological space, recall that extension $\tau^{\mathrm{i}}[6]$ is the family of all $i$-open subsets of space $X$.

Remark 1.9. [6] $\left(\mathrm{X}, \tau^{\mathrm{i}}\right)$ need not to be a topological space.

Definition 1.10. [6] A topological space $(X, \tau)$ is said to be Topologically Extended for i-open sets (shortly T.E.I) if and only if $\left(\mathrm{X}, \tau^{\mathrm{i}}\right)$ is a topological space. Otherwise is called not T.E.I. 
Theorem 1.11. [6] Let $\mathrm{X}$ be a non-empty finite set and let $\tau=\{\phi, A, X\}$ where, $A$ is a subset of $X$ and containing only one element. Then, $(X, \tau)$ is T.E.I. (i.e. $\left(X, \tau^{i}\right)$ is a topological space).

Corollary 1.12. Let $\left(X, \tau_{1}, \tau_{2}\right)$ be a bitopological space and let $\left(X, \tau_{1}\right)$ be a (T.E.I.) topological space as like as in Theorem 1.11 , let $\tau_{2}=\tau_{1}^{i}$ where, $\tau_{1}^{i}$ is the family of all $\mathrm{i}$ open sets in a topological space $\left(X, \tau_{1}\right)$, then,. $=\tau_{2} i$-open sets $\tau_{1} \tau_{2}-$

Proof Suppose that $X=\left\{x_{1}, x_{2}, \ldots \ldots \ldots, x_{n}\right\}$ and $\tau_{1}=\left\{\phi,\left\{x_{1}\right\}, X\right\}$.

$\tau_{1}$-open sets are $: \phi,\left\{x_{1}\right\}, X$.

By definition of i-open sets, we have:

$\tau_{1}^{i}=\left\{\phi,\left\{x_{1}\right\},\left\{x_{1}, x_{2}\right\},\left\{x_{1}, x_{3}\right\}, \ldots \ldots \ldots,\left\{x_{1}, x_{n}\right\},\left\{x_{1}, x_{2}, x_{3}\right\},\left\{x_{1}, x_{2}, x_{4}\right\}, \ldots \ldots\right.$,

$\left.\left\{x_{1}, x_{2}, x_{n}\right\}, \ldots \ldots \ldots \ldots,\left\{x_{1}, x_{3}, x_{4}, \ldots \ldots, x_{n}\right\},\left\{x_{1}, x_{2}, \ldots \ldots \ldots, x_{n}\right\}=X\right\}$.

Since, $\tau_{2}=\tau_{1}^{i}$ then $\tau_{2}$-closed sets are $:\left\{x_{1}, x_{2}, \ldots, x_{n}\right\}=X$,

$\left\{x_{2}, x_{3}, x_{4}, \ldots, x_{n}\right\},\left\{x_{3}, x_{4}, \ldots, x_{n}\right\},\left\{x_{2}, x_{4}, \ldots, x_{n}\right\}, \ldots \ldots,\left\{x_{2}, \ldots, x_{n-1}\right\},\left\{x_{4}, \ldots, x_{n}\right\}$,

$\left\{x_{3}, x_{5}, . ., x_{n}\right\}, \ldots,\left\{x_{3}, \ldots, x_{n-1}\right\}, \ldots,\left\{x_{2}\right\}, \phi$.

Since, $\left\{x_{1}\right\}$ is the alone $\tau_{1}$-open set $\neq \phi, X$ and the intersection between $\left\{x_{1}\right\}$ and the sets $\left\{x_{2}\right\},\left\{x_{3}\right\}, \ldots,\left\{x_{n}\right\}, \ldots,\left\{x_{2}, x_{3}\right\}, \ldots,\left\{x_{2}, x_{n}\right\},\left\{x_{2}, x_{3}, x_{4}\right\}, \ldots$.

$\left\{x_{2}, x_{3}, x_{n}\right\}, \ldots,\left\{x_{3}, x_{4}, x_{n}\right\}, \ldots,\left\{x_{n-2}, x_{n-1}, x_{n}\right\}$ which does not contain $\left\{x_{1}\right\}$, equal to $\phi$ and by the same way in Theorem 1.11 we have:

$\tau_{1} \tau_{2}-i$-opensets $=\tau_{2}$ where, $\tau_{2}=\tau_{1}^{i}$

Example 1.13. Let $X=\{a, b, c\}$,

$\tau_{1}=\{\phi,\{a\}, X\}, \tau_{2}=\tau_{1}^{i}=\{\phi,\{a\},\{a, b\},\{a, c\}, X\}$

$\tau_{2}-$ closed sets are $: \phi,\{b, c\},\{c\},\{b\}, X$.

By the same way of the examples mentioned above, we have:

$\tau_{1} \tau_{2}-i-$ opensets $=\tau_{2}$

Definition 1.14. A set $A$ of a bitopological space $\left(X, \tau_{1}, \tau_{2}\right)$ is called:

1. $\tau_{1} \tau_{2}-$ generalized closed set $\left(\tau_{1} \tau_{2}-g-\right.$ closed set $)$ [3]

if $\tau_{2}-C l(A) \subseteq U$ where $A \subseteq U$ and $U \subseteq X$ is $\tau_{1}$-open set.

2. $\tau_{1} \tau_{2}-g$-open set [3] if $X-A$ is $\tau_{1} \tau_{2}-g-$ closed.

3. $\tau_{1}$-closed set.is $\subseteq X F \subseteq A$ where $F \subseteq \tau_{2}-\operatorname{Int}_{i}(A)$ if $\tau_{1} \tau_{2}-g i$-open set

4. $\tau_{1} \tau_{2}-g i$-open. is $X-A$ if $\tau_{1} \tau_{2}-g i$-closed set

5. $\tau_{1} \tau_{2}-i-$ star genralzed closed set $\left(\tau_{1} \tau_{2}-i * g-\right.$ closed set $)$

if $\tau_{2}-C l(A) \subseteq U$ where $A \subseteq U$ and $U \subseteq X$ is $i$-openset. $\tau_{1}-$

6. $\tau_{1} \tau_{2}-i$-star genralzed openset $\quad\left(\tau_{1} \tau_{2}-i * g-\right.$ open set $) \quad$ if $\quad X-A \quad$ is $\tau_{1} \tau_{2}-i * g-$ closed

7. $\tau_{1} \tau_{2}-$ genralzed $w$-closed set $\left(\tau_{1} \tau_{2}-g w-\right.$ closed set $)[1]$

if $\tau_{2}-C l_{w}(A) \subseteq U$ where $A \subseteq U$ and $U \subseteq X$ is $\tau_{1}$-open set.

8. $\tau_{1} \tau_{2}-$ genralzed $w$-open set $\left(\tau_{1} \tau_{2}-g w\right.$-open set $)[1] \quad$ if $\quad X-A \quad$ is $\tau_{1} \tau_{2}-g w-$ closed 
In the following example $X$ is a finite set.

Example 1.15. Let $X=\{a, b, c\}, \tau_{1}=\{\phi,\{a\}, X\} \tau_{2}=\{\phi,\{a\}, X\}$.

From definitions mentioned above we have:

$\tau_{1}$-open sets $: \phi,\{a\}, X, \tau_{1}-$ closed sets $: \phi,\{b, c\}, X$.

$\tau_{1}-w$-closed sets $: \phi,\{a\},\{b\},\{c\},\{a, b\},\{a, c\},\{b, c\}, X$.

$\tau_{1}-w$-open sets $: \phi,\{a\},\{b\},\{c\},\{a, b\},\{a, c\},\{b, c\}, X$.

$\tau_{1}-i$-open sets $: \phi,\{a\},\{a, b\},\{a, c\}, X$.

$\tau_{1}-i$-closed sets $: \phi,\{b, c\},\{c\},\{b\}, X$.

$\tau_{2}-$ open sets $: \phi,\{a\}, X, \tau_{2}-$ closed sets $: \phi,\{b, c\}, X$.

$\tau_{2}-w$-closed sets $: \phi,\{a\},\{b\},\{c\},\{a, b\},\{a, c\},\{b, c\}, X$.

$\tau_{2}-w$-open sets $: \phi,\{a\},\{b\},\{c\},\{a, b\},\{a, c\},\{b, c\}, X$.

$\tau_{2}-i$-open sets $: \phi,\{a\},\{a, b\},\{a, c\}, X$.

$\tau_{2}-i-$ closed sets $: \phi,\{b, c\},\{c\},\{b\}, X$.

$\tau_{1} \tau_{2}-g$-closed sets $: \phi,\{b\},\{c\},\{a, b\},\{a, c\},\{b, c\}, X$.

$\{a\}$ is not $\tau_{1} \tau_{2}-g$-closed set because $\tau_{2}-C l(\{a\})=X \subseteq X$

but $\tau_{2}-C l(\{a\})=X \not \subset\{a\}($ definition(1.14)(1)) .

$\tau_{1} \tau_{2}-g$-open sets $: \phi,\{a, c\},\{a, b\},\{c\},\{b\},\{a\}, X$.

but $\{b, c\}$ is not $\tau_{1} \tau_{2}-g$-open set because $\{a\}^{C}=\{b, c\}$

and $\{a\}$ is not $\tau_{1} \tau_{2}-g$-closed set(difinition1.14(2))

$\tau_{1} \tau_{2}-$ gi-open sets $: \phi, X, \tau_{1} \tau_{2}-$ gi-closed sets $: \phi, X$.

$\tau_{1} \tau_{2}-i^{*} g-$ closed sets $: \phi,\{b, c\}, X, \tau_{1} \tau_{2}-i^{*} g-$ open sets $: \phi,\{a\}, X$.

$\tau_{1} \tau_{2}-g w$-closed sets $: \phi,\{a\},\{b\},\{c\},\{a, b\},\{a, c\},\{b, c\}, X$.

$\tau_{1} \tau_{2}-g w-$ open sets $: \phi,\{a\},\{b\},\{c\},\{a, b\},\{a, c\},\{b, c\}, X$.

\section{In the following example $X$ is an infinite set.}

Example 1.16. Let $X=R, \tau_{1}=\{\phi, R-Q, R\} \tau_{2}=\{\phi, Q, R\}$. Where, $R$ is the set of real numbers, $Q$ is the set of rational numbers and $R-Q$ is the set of irrational numbers.

From definitions mentioned above, we have:

$\tau_{1}$-open sets $: \phi, R-Q, R, \tau_{1}-$ closed sets $: \phi, Q, R$.

$\tau_{1}-w$-closed sets $: \phi, R-Q, Q, R$, other sets $\subseteq R$ which satisfies the

definition of $w$-closed sets.

$\tau_{1}-w$-open sets $: \phi, R-Q, Q, R$, other sets $\subseteq R$ which are the

complements of $\tau_{1}-w$-closed sets.

$\tau_{1}-i$-open sets $: \phi, R-Q, R$, other sets $\subseteq R$ which it satisfies the

definition of $i$-open sets. $Q$ is not $\tau_{1}-i$-open set.

$\tau_{1}-i$-closed sets $: \phi, Q, R$, other sets $\subseteq R$ which are the

complements of $\tau_{1}-i$-open sets. $R-Q$ is not $\tau_{1}-i-$ closed set.

$\tau_{2}$ - open sets $: \phi, Q, R, \tau_{2}-$ closed sets $: \phi, R-Q, R$.

$\tau_{2}-w$-closed sets $: \phi, R-Q, Q, R$, other sets $\subseteq R$ which it satisfies the

definition of $w$-closed sets. 
$\tau_{2}-w$-open sets $: \phi, R-Q, Q, R$, other sets $\subseteq R$ which are the complements of $\tau_{2}-w$-closed sets.

$\tau_{2}-i$-open sets $: \phi, Q, R$, other sets $\subseteq R$ which it satisfies the definition of $i$-open sets.

$\tau_{2}-i$-closed sets $: \phi, R-Q, R$, other sets $\subseteq R$ which are the complements of $\tau_{2}-i$-open sets.

$\tau_{1} \tau_{2}-g-$ closed sets $: \phi, R-Q, Q, R$, other sets $\subseteq R$ which it satisfies the definition of $\tau_{1} \tau_{2}-g-$ closed sets.

$\tau_{1} \tau_{2}-g$-open sets $: \phi, R-Q, Q, R$, other sets $\subseteq R$ which are the complements of $\tau_{1} \tau_{2}-g-$ closed sets.

$\tau_{1} \tau_{2}-$ gi-open sets $: \phi, Q, R$,other sets $\subseteq R$ which it satisfies the definition of $\tau_{1} \tau_{2}-$ gi-open sets. $R-Q$ is not $\tau_{1} \tau_{2}-$ gi-open set. $\tau_{1} \tau_{2}-$ gi-closed sets $: \phi, R-Q, R$, other sets $\subseteq R$ which are the complements of $\tau_{1} \tau_{2}-$ gi-open sets. $Q$ is not $\tau_{1} \tau_{2}-$ gi-closed set. $\tau_{1} \tau_{2}-i^{*} g$-closed sets $: \phi, R-Q, Q, R$, other sets $\subseteq R$ which it satisfies the definition of $\tau_{1} \tau_{2}-i * g-$ closed sets.

$\tau_{1} \tau_{2}-i * g$-open sets $: \phi, R-Q, Q, R$, other sets $\subseteq R$ which are the complements of $\tau_{1} \tau_{2}-i * g-$ closed sets.

$\tau_{1} \tau_{2}-g w-$ closed sets $: \phi, R-Q, Q, R$, other sets $\subseteq R$ which it satisfies the definition of $\tau_{1} \tau_{2}-g w-$ closed sets.

$\tau_{1} \tau_{2}-g w$-open sets $: \phi, R-Q, Q, R$, other sets $\subseteq R$ which are the complements of $\tau_{1} \tau_{2}-g w-$ closed sets.

\section{2. i-Star Generalized w-Closed and i-Star Generalized w-Open Sets in Bitopological Spaces.}

Throughout this section, we define i-star generalized w-closed, i-star generalized w-open sets and study their basic properties in bitopological spaces.

Definition 2.1. A set $A$ of a bitopological space $\left(X, \tau_{1}, \tau_{2}\right)$ is said to be $\tau_{1} \tau_{2}-i-$ star genralized $w-$ closed set $\left(\tau_{1} \tau_{2}-i * g w-\right.$ closed set $)$, if $\tau_{2}-C l_{w}(A) \subseteq U$ where, $A \subseteq U$ and $U \subseteq X$ is a $\tau_{1}-i$-openset.

In Example 1.15, we have: $\tau_{1} \tau_{2}-i^{*} g w-$ closed sets $: \phi,\{a\},\{b\},\{c\},\{a, b\},\{a, c\},\{b, c\}, X$. in Example 1.16, we have: $\tau_{1} \tau_{2}-i^{*} g w-$ closed sets $: \phi, R-Q, Q, R$, other sets $\subseteq R$ which it satisfies the definition of $\tau_{1} \tau_{2}-i * g w-$ closed sets.

Remark 2.2. [6] Every open set in a topological space $(X, \tau)$ is i-open .

Theorem 2.3. Let $\left(X, \tau_{1}, \tau_{2}\right)$ be a bitopological space and $A \subseteq X$ then, the followings are true:

1. If $A$ is $\tau_{2}-w$-closed then, $A$ is $\tau_{1} \tau_{2}-i^{*} g w-$ closed. 
2. If $A$ is $\tau_{1}-i-o p e n$ and $\tau_{1} \tau_{2}-i * g w-$ closed then, $A$ is $\tau_{2}-w-$ closed.

3. If $A$ is $\tau_{1} \tau_{2}-i * g w-$ closed then, $A$ is $\tau_{1} \tau_{2}-g w-$ closed .

\section{Proof}

1. Suppose that $A$ is $i$-open. $\tau_{1}$-are $U \subseteq X$ and $A \subseteq U$. Let $\tau_{2}-w$-closed then $\tau_{2}-C l_{w}(A)=A \subseteq U$.

Therefore, $A$ is $\tau_{1} \tau_{2}-i * g w-$ closed .

2. Suppose that $A$ is $\tau_{1}-i$-open and $\tau_{1} \tau_{2}-i^{*} g w-$ closed. Let $A \subseteq A$ and $A$ is $\tau_{1}-i$-open. Then, $\tau_{2}-C l_{w}(A) \subseteq A$. Therefore, $\tau_{2}-C l_{w}(A)=A$. Then, $A$ is $\tau_{2}-w$-closed .

3. Suppose that $A$ is $\tau_{1} \tau_{2}-i^{*} g w-$ closed. Let $A \subseteq U$ and. Since, $\tau_{1}-$ open is $U \subseteq X$

is $\quad A$. Then, $\tau_{2}-C l_{w}(A) \subseteq U\left(\right.$ Remark 2.2), we have $X$ in $i$-open $\tau_{1}-$ is $U$ $\tau_{1} \tau_{2}-g w-$ closed

Theorem 2.4. Let $\left(X, \tau_{1}, \tau_{2}\right)$ be a bitopological space, then every $\tau_{1} \tau_{2}-i * g-$ closed set in $X$ is $\tau_{1} \tau_{2}-i * g w-$ closed .

Proof Suppose that $A$ is $\tau_{1} \tau_{2}-i * g-$ closed set, we have $\tau_{2}-C l(A) \subseteq U$, where $A \subseteq U$ and $U \subseteq X$ are $\tau_{1}-i$-open set .

Since, $\tau_{2}-C l_{w}(A) \subseteq \tau_{2}-C l(A)$,

we have $\tau_{2}-C l_{w}(A) \subseteq \tau_{2}-C l(A) \subseteq U$.

Therefore, $A$ is $\tau_{1} \tau_{2}-i^{*} g w-$ closed

Remark 2.5. The converse of Theorem 2.4 is not true. Indeed, in Example 1.15, $A=\{\mathrm{a}, \mathrm{b}\}$ is $\tau_{1} \tau_{2}-i * g w-$ closed set, but is not $\tau_{1} \tau_{2}-i * g-$ closed .

$$
\tau_{1} \tau_{2}-i^{*} g-\text { closed set } \tau_{1} w-\text { closed }
$$

Theorem 2.6. If $A$ is $\tau_{1} \tau_{2}-i * g w-$ closed set in $X$ and $A \subseteq B \subseteq \tau_{2}-C l_{w}(A)$, then $B$ is $\tau_{1} \tau_{2}-i * g w-$ closed set .

Proof Suppose that $A$ is $\tau_{1} \tau_{2}-i * g w-$ closed set in $X$ and $A \subseteq B \subseteq \tau_{2}-C l_{w}(A)$. Let $B \subseteq U$ and $U$ is $\tau_{1}-i$-open set. Then, $A \subseteq U$.Since, $A$ is $\tau_{1} \tau_{2}-i * g w-$ closed set ,we have $\tau_{2}-C l_{w}(A) \subseteq U$. Since, $B \subseteq \tau_{2}-C l_{w}(A), \tau_{2}-C l_{w}(B) \subseteq \tau_{2}-C l_{w}(A) \subseteq U$. Hence, $B$ is $\tau_{1} \tau_{2}-i * g w-$ closed .

Theorem 2.7. If $A$ and $B$ are $\tau_{1} \tau_{2}-i^{*} g w-$ closed sets then, so is $A \cup B$.

Proof Suppose that $A$ and $B$ are $\tau_{1} \tau_{2}-i^{*} g w$-closed sets. Let $U \subseteq X$ be $\tau_{1}-i-$ open set and $(A \cup B) \subseteq U$. Then, $A \subseteq U$ and $B \subseteq U$. Since, $A$ and $\boldsymbol{B}$ are $\tau_{1} \tau_{2}-i * g w-$ closed sets, we have $\tau_{2}-C l_{w}(A) \subseteq U$ and $\tau_{2}-C l_{w}(B) \subseteq U$. Then, $\tau_{2}-C l_{w}(A \cup B) \subseteq U$. Therefore, $A \cup B$ is $\tau_{1} \tau_{2}-i^{*} g w-$ closed set.

Theorem 2.8. Let $\left(X, \tau_{1}, \tau_{2}\right)$ be a bitopological space and $A \subseteq X$ then, the following are true: 
1. If $A$ is $\tau_{2}$-closed then, $A$ is $\tau_{2}-w$-closed.

2. If $A$ is $\tau_{1} \tau_{2}-i * g-$ closed then,. $\tau_{1} \tau_{2}-g-$ closed is $A$

3. If $A$ is $\tau_{1} \tau_{2}-g$-closed then, $A$ is $\tau_{1} \tau_{2}-g w-$ closed .

\section{Proof}

1. Suppose that $A$ is $\tau_{2}$-closed. Then $\tau_{2}-C l(A)=A$. Since, $\tau_{2}-C l_{w}(A) \subseteq \tau_{2}-C l(A)=A$, we have $\tau_{2}-C l_{w}(A)=A$. Therefore, $A$ is $\tau_{2}-w-$ closed .

2. Suppose that $A$ is $A \subseteq U$. Let $\tau_{1} \tau_{2}-i^{*} g$-closed and. $\tau_{1}-$ open is $U \subseteq X$ Therefore, $\tau_{2}-C l(A) \subseteq U$. Then, $A$ is $\tau_{1} \tau_{2}-g$-closed .

3. Suppose that $A$ is $\tau_{1} \tau_{2}-g$-closed. Let $A \subseteq U$ and. $\tau_{1}$-open are $U \subseteq X$ Therefore, $\tau_{2}-C l(A) \subseteq U$.

Since $\tau_{2}-C l_{w}(A) \subseteq \tau_{2}-C l(A) \subseteq U$, we have $\tau_{2}-C l_{w}(A) \subseteq U$. Then, $A$ is $\tau_{1} \tau_{2}-g w-$ closed

Remark 2.9. The converses of Theorem 2.8 are not true. Indeed, In Example 1.15, $A$ $=\{a, c\}$ is $\tau_{2}-w$-closed set, but is not $\tau_{2}-$ closed, $A=\{a, c\}$ is $\tau_{1} \tau_{2}-g$-closed set, but is not $\tau_{1} \tau_{2}-i * g-$ closed set. Also $\{\mathrm{a}\}$ is $\tau_{1} \tau_{2}-g w-$ closed set but, is not $\tau_{1} \tau_{2}-g-$ closed .

$$
\tau_{1} \tau_{2}-i * g-\text { closed set } \tau_{1} \tau_{2}-\text { closed set } \tau_{2}-w
$$

Definition 2.10. A set $A$ of a bitopological space $\left(X, \tau_{1}, \tau_{2}\right)$ is said to be $\tau_{1} \tau_{2}-i-$ star genralzed $w$-open set $\left(\tau_{1} \tau_{2}-i * g w-o p e n\right.$ set $)$, if $X-A$ is $\tau_{1} \tau_{2}-i * g w-$ closed set.

In Example 1.15. we have: $\tau_{1} \tau_{2}-i * g w-$ open sets : $\phi,\{a\},\{b\},\{c\},\{a, b\},\{a, c\},\{b, c\}, X$.

Also, in Example 1.16 we have:

$\tau_{1} \tau_{2}-i * g w-$ open sets $: \phi, R-Q, Q, R$, other sets $\subseteq R$ which it satisfies the definition of $\tau_{1} \tau_{2}-i * g w-o p e n$ sets.

Theorem 2.11. A set $A$ is $\tau_{1} \tau_{2}-i * g w-o p e n$ set if and only if $F \subseteq \tau_{2}-\operatorname{Int}_{w}(A)$, where $F \subseteq A$ and $F \subseteq X$ is. $i$-closed set $\tau_{1}-$

Proof Suppose that $A$ is $\tau_{1} \tau_{2}-i * g w$-open set. Suppose that $F \subseteq X$ is $\tau_{1}-$ $F^{C}$. Then $F \subseteq A$ and $i$-closed set is is $A^{C}$. Since, $A^{C} \subseteq F^{C}$ and $i$-open $\tau_{1}-$ 
Since, $\tau_{2}-C l_{w}\left(A^{C}\right) \subseteq F^{C}, \quad$ we $\quad$ have $\quad \tau_{1} \tau_{2}-i * g w-$ closed set $. F \subseteq \tau_{2}-\operatorname{Int}_{w}(A)$, we have $\tau_{2}-C l_{w}\left(A^{C}\right)=\left[\tau_{2}-\operatorname{Int}_{w}(A)\right]^{C}$

Conversely, suppose that $F \subseteq \tau_{2}-\operatorname{Int}_{w}(A)$ where $F \subseteq A$ and $F \subseteq X$ is $\tau_{1}-$ $F^{C}$ and $\quad A^{C} \subseteq F^{C}$. Then, $i$-closed set is and $F \subseteq \tau_{2}-\operatorname{Int}_{w}(A)$. Since, $i$-open $\tau_{1}-$ is $A^{C}$. Then, $\quad \tau_{2}-C l_{w}\left(A^{C}\right) \subseteq F^{C}, \quad$ we have $\tau_{2}-C l_{w}\left(A^{C}\right)=\left[\tau_{2}-\operatorname{Int}_{w}(A)\right]^{C}$ $\tau_{1} \tau_{2}-i * g w-$ closed set. Therefore, $\square \quad \tau_{1} \tau_{2}-i * g w-$ open set is $A$

Theorem 2.12. If $A$ and $B$ are separated $\tau_{1} \tau_{2}-i^{*} g w-$ open sets, then so is $A \cup B$.

Proof Suppose that $A$ and $B$ are $\tau_{1} \tau_{2}-i * g w$-opensets. Let $F \subseteq X$ be $\tau_{1}-i$-closed set and $F \subseteq(A \cup B)$. Since $A$ and $B$ are separated sets, we have $\tau_{1}-C l(A) \cap B=A \cap \tau_{1}-C l(B)=\phi$.

Also, $\tau_{2}-C l(A) \cap B=A \cap \tau_{2}-C l(B)=\phi$

Then, $F \cap \tau_{2}-C l(A) \subseteq(A \cup B) \cap \tau_{2}-C l(A)=A$. By the same way, we have $F \cap \tau_{2}-C l(B) \subseteq B$. Since, $F \subseteq X$ is $\tau_{1}-i-$ closed set, we have $F \cap \tau_{1}-C l(A)$ and $F \cap \tau_{1}-C l(B)$ are $\tau_{1}-i$-closed sets. Since, $A$ and $B$ are $\tau_{1} \tau_{2}-i * g w$-open sets, we have $F \cap \tau_{2}-C l(A) \subseteq \tau_{2}-\operatorname{Int}_{w}(A)$ and $F \cap \tau_{2}-C l(B) \subseteq \tau_{2}-\operatorname{Int}_{w}(B)$. Now

$$
\begin{aligned}
F=F \cap(A \cup B) & \subseteq\left(F \cap \tau_{2}-C l(A)\right) \cup\left(F \cap \tau_{2}-C l(B)\right) \\
& \subseteq \tau_{2}-I_{w}(A \cup B) .
\end{aligned}
$$

Therefore, $A \cup B$ is $\tau_{1} \tau_{2}-i * g w-$ open set.

Theorem 2.13. If $A$ and $B$ are $\tau_{1} \tau_{2}-i * g w-o p e n s e t s$ then so is $A \cap B$.

Proof Suppose that $A$ and $B$ are $\tau_{1} \tau_{2}-i * g w$-opensets. Let $F \subseteq X$ be $\tau_{1}-i-$ closed set and $F \subseteq(A \cap B)$, we have $F \subseteq A$ and $F \subseteq B$. Since, $A$ and $B$ are $\tau_{1} \tau_{2}-i * g w-o p e n s e t s$, we have $F \subseteq \tau_{2}-\operatorname{Int}_{w}(A)$ and $F \subseteq \tau_{2}-\operatorname{Int}_{w}(B)$. Then $F \subseteq \tau_{2}-\operatorname{Int}_{w}(A \cap B)$.

Therefore, $A \cap B$ is $\tau_{1} \tau_{2}-i * g w-o p e n$ set.

Theorem 2.14. If $A$ is $\tau_{1} \tau_{2}-i * g w-o p e n s e t$ in $X$ and $\tau_{2}-\operatorname{Int}_{w}(A) \subseteq B \subseteq A$, then $\boldsymbol{B}$ is $\tau_{1} \tau_{2}-i * g w-$ open set .

Proof Suppose that $A$ is $\tau_{1} \tau_{2}-i * g w-o p e n$ set in $X$ and $\tau_{2}-\operatorname{Int}_{w}(A) \subseteq B \subseteq A$. Let $F \subseteq X$ be $\tau_{1}-i$-closed set and $F \subseteq B$. Since, $F \subseteq B$ and $B \subseteq A$, we have $F \subseteq A$. Since, $A$ is $\tau_{1} \tau_{2}-i * g w-o p e n s e t$, we have $F \subseteq \tau_{2}-\operatorname{Int}_{w}(A)$ and Since, $\tau_{2}-\operatorname{Int}_{w}(A) \subseteq B$, we have $\tau_{2}-\operatorname{Int}_{w}(A) \subseteq \tau_{2}-\operatorname{Int}_{w}(B)$. Then, $F \subseteq \tau_{2}-\operatorname{Int}_{w}(B)$. Therefore, $\boldsymbol{B}$ is $\tau_{1} \tau_{2}-i * g w-o p e n$.

Theorem 2.15. Let $\left(X, \tau_{1}, \tau_{2}\right)$ be a bitopological space and $A \subseteq X$ then the followings are true:

1. If $A$ is $\tau_{2}-w$-open, then $A$ is $\tau_{1} \tau_{2}-i^{*} g w-o p e n$.

2. If $A$ is $\tau_{1}-i$-closed and $\tau_{1} \tau_{2}-i * g w-o p e n$, then $A$ is $\tau_{2}-w$-open.

3. If $A$ is $\tau_{1} \tau_{2}-i^{*} g w-o p e n$ then $A$ is $\tau_{1} \tau_{2}-g w-o p e n$. 
4. If $A$ is $\tau_{1} \tau_{2}-i * g-o p e n$ then $A$ is $\tau_{1} \tau_{2}-i * g w-o p e n$.

5. If $A$ is $\tau_{2}$-open then $A$ is $\tau_{2}-w$-open . [3]

6. If $A$ is $\tau_{1} \tau_{2}-i * g$-open then $A$ is $\tau_{1} \tau_{2}-g-o p e n$.

7. If $A$ is $\tau_{1} \tau_{2}-g$-open then $A$ is $\tau_{1} \tau_{2}-g w-o p e n$.

\section{Proof}

1. Suppose that $A$ is $\tau_{2}-w$-open. We have $A^{C}$ is $\tau_{2}-w$-closed. Then,is $A^{C}$ (Theorem2.3(1)) $. \tau_{1} \tau_{2}-i * g w-$ closed

Therefore, $A$ is $\tau_{1} \tau_{2}-i^{*} g w-o p e n$.

2. Suppose that $A$ is $\tau_{1}-i-$ closed and $\tau_{1} \tau_{2}-i^{*} g w-o p e n$. Then, $A^{C}$ is $\tau_{1}-i$-open and $\tau_{1} \tau_{2}-i * g w-$ closed

Then,. $\tau_{2}-w$-open is $A$ (Theorem2.3(2)). Therefore, $\tau_{2}-w$ closed is $A^{C}$

3. Suppose that $A$ is $\tau_{1} \tau_{2}-i^{*} g w-o p e n$. Then, $A^{C}$ is $\tau_{1} \tau_{2}-i^{*} g w-$ closed, hence $A^{C}$ is $\tau_{1} \tau_{2}-g w-$ closed (Theorem2.3(3)). Therefore, $A$ is $\tau_{1} \tau_{2}-g w-o p e n$.

4. Suppose that $A$ is $\tau_{1} \tau_{2}-i^{*} g$-open. Then, $A^{C}$ is $\tau_{1} \tau_{2}-i^{*} g$-closed, hence $A^{C}$ is $\tau_{1} \tau_{2}-i * g w-$ closed (Theorem 2.4). Therefore, $A$ is $\tau_{1} \tau_{2}-i * g w-o p e n$.

5. (see [3]).

6. Suppose that $A$ isis $A^{C}$, hence $\tau_{1} \tau_{2}-i^{*} g-$ closed is $A^{C}$. Then, $\tau_{1} \tau_{2}-i^{*} g-$ open $\tau_{1} \tau_{2}-g-$ closed (Theorem 2.8(2))

Therefore, $A$ is $\tau_{1} \tau_{2}-g$-open .

7. Suppose that $A$ is $\tau_{1} \tau_{2}-g$-open. Then, $A^{C}$ is $\tau_{1} \tau_{2}-g$-closed, hence $A^{C}$ is $\tau_{1} \tau_{2}-g w-$ closed (Theorem 2.8(3)).

Therefore, $A$ is $\tau_{1} \tau_{2}-g w-o p e n$.

Remark 2.16. The converses of Theorem 2.15(4)(5)(6)(7) are not true. Indeed, In Example $1.15, A=\{b\}$ is $\tau_{1} \tau_{2}-i * g w-o p e n$, but it is not $=A$ and $\tau_{1} \tau_{2}-i * g-o p e n$ $\{b\}$ is $\tau_{2}-w$-open set, but it is not $\tau_{2}$-open. Also, $A=\{b\}$ is $\tau_{1} \tau_{2}-g$-open set, but it is not $\tau_{1} \tau_{2}-i * g-$ open set .

$\{b, c\}$ is $\tau_{1} \tau_{2}-g w-o p e n$ set but it is not $\tau_{1} \tau_{2}-g-$ open .

$$
\tau_{1} \tau_{2}-i * g-\text { open set } \tau_{1} \tau_{2}
$$




\section{REFERENCES}

[1] Al- Zoubi, K. Y.,(2005),"On generalized w-closed sets", Int. J. of Mathematics and Math. Sciences, 13.

[2] Benchalli, S.S., Patil, P.G. and Rayanagoudar, T.D.,(2010), "On w-Locally Closed Sets in Bitopological Spaces", Int. Journal of Math. Analysis, Vol.4, no.39, 19371944.

[3] Chandrasekhara Rao, K. and Narasemhan, D. ,(2009), "Semi Star Generalized wclosed sets in Bitopological spaces", Int. J. Contemp. Math. Sciences, Vol.4. no.12, 587-595.

[4] Chandrasekhara Rao, K. and Kannan, K. ,(2006), "Regular Star Generalized Closed Sets in Bitopological Spaces", Thai J. of Math., Vol. 4. no. 2, 341-349.

[5] Mahdi, Y.K.,(2007), "Semi-open and Semi-closed sets in Bitopological spaces", accepted in the first science Conference of education college , Babylon Univ., February 18-19.

[6] Mohammed, A.A. and Askandar, S.W. ,(2012), "On i-open sets", UAE Math Day Conference, American Univ. of Sharjah, April 14.

[7] Nagaveni, N. and Rajarubi, P. ,(2012), "GRW-Closed Sets and GRW-Continuity in Bitopological spaces", European Journal of Scientific Research, ISSN 1450-216X Vol.78 no.1, pp.5-14.

[8] Sheik John, M. and Maragathavalli, S. ,(2010), "Strongly $\alpha g *$-closed sets in Bitopological spaces", Int. J. Contemp. Math. Sciences, Vol.5. no.17, 805-813.

[9] Sheik John, M. and Sundaram, P. ,(2004)," $g$ *-Closed Sets in Bitopological Spaces", Indian J. Pure appl. Math., 35(1): 71-80, January. 